\title{
Maternal, Fetal and Neonatal Outcomes in Mothers With Diabetes Mellitus or Gestational Diabetes That Complicated With Preterm Premature Rupture of the Membrane (PPROM)
}

\author{
Aila Kari ${ }^{1}$, Farnaz Sahhaf ${ }^{1 *}$, Fatemeh Abbasalizadeh ${ }^{1}$
}

\begin{abstract}
Objectives: It has been suggested that the presence of diabetes may increase the possibility of occurrence of premature rupture of the membrane (PPROM), which in turn, could lead to a dramatic escalation in the rate of pregnancy-related complications. The available data, however, are not sufficient in this regard in the literature. This study aims to examine pregnancy outcome in pregnant women with simultaneous diabetes mellitus/gestational diabetes and PPROM.

Materials and Methods: A total of 134 pregnant women with gestational diabetes $(n=99)$ or diabetes mellitus $(n=35)$ were compared with 135 pregnant women normoglycemic women in terms of maternal, fetal and neonatal outcomes in a teaching hospital. Multiplepregnancy, anomalous fetus, complicated pregnancies, polyhydramnios, placental problems, and intrauterine growth restriction were exclusion criteria.

Results: The three groups were comparable for the incidence of cesarean section, labor induction, chorioamnionitis, postpartum hemorrhage, need for ICU/NICU admission, placental retention, transfusion requirement, neonatal infection, respiratory distress, need for resuscitation, icterus, dystocia, need for insulin injection, and maternal/fetal mortality, as well as the mean Apgar and neonatal hospital stay. The mean maternal hospital stay and the duration of vaginal delivery were significantly shorter in controls than in patients with gestational diabetes. The rate of neonatal hypoglycemia was significantly higher in the group with diabetes mellitus compared to that in the two other groups.

Conclusion: Except for the maternal hospital stay, duration of vaginal delivery and the incidence of neonatal hypoglycemia that were significantly better in normoglycemic mothers, the three groups were comparable for the remaining outcome variables.

Keywords: Diabetes mellitus, Gestational diabetes, Premature rupture of the membrane
\end{abstract}

\section{Introduction}

Preterm birth is the main cause of neonatal morbidity and mortality, which is divided into three categories: preterm premature rupture of membrane (PPROM), preterm labor, and early delivery resulting from medical intervention. PPROM is defined as a condition in which the amniotic membranes are ruptured before 37 weeks of gestation and before the onset of labor, while the extreme PPROM occurs before 26 weeks of gestation (1). PPROM is associated with several risks for both mothers and fetuses. The most important maternal complication of PPROM is intra-amniotic infection (chorioamnionitis), which may affect fetus negatively, as well. Other fetal complications of PPROM are placental abruption, fetal distress, fetal restrictive deformities, pulmonary hypoplasia, preterm birth and fetal or newborn death (2-4). Diabetes mellitus or gestational diabetes is a complication which may be associated with PPROM outbreak or exacerbation during pregnancy. Diabetes mellitus is featured with hyperglycemia and impaired metabolism of carbohydrates, fatty acids and proteins and is associated with the absolute or relative lack of insulin. It is amongst the most common and costly diseases throughout the world whose prevalence rate is increasing because of the changed lifestyles and the improved medical and sanitary conditions, which in turn have enhanced the survival rate in the societies (5). In Iran, the general prevalence rate of diabetes and impaired glucose tolerance (IGT) in people of childbirth age is estimated about $7.6 \%$ (6).

As the previous studies demonstrated, there is a direct relationship between (mellitus and gestational) diabetes and PPROM in pregnant women and unpleasant consequences of pregnancy (7-15).

Given the considerable prevalence rate of (mellitus and gestational) diabetes during the childbirth age and the high possibility of infection and other neonatal and fetal complications in such pregnancies, which are exacerbated by PPROM, and since there has not been found any study covering maternal, neonatal and fetal complications in diabetic pregnant women with PPROM simultaneously (including in Iran), this study tries to consider maternal, fetal and neonatal complications of PPROM in women 
with (mellitus or gestational) diabetes.

\section{Methods and Materials}

In this descriptive-analytical and cross-sectional study, women at 24-34 weeks of gestation who have experienced preterm premature rupture of membrane (PPROM) were grouped into three groups, diabetes-free, with gestational diabetes and with diabetes mellitus and then were compared in terms of gestational consequences. The study was conducted at Tabriz Al-Zahra medical and educational center. The study's length was considered 15 months (December, 2014 to March, 2015); however, it must be noted that in some cases the study was continued retrospectively by 2006 . Regarding the retrospective nature of a great part of the study and lack of an interventional measure in both diagnosis and treatment procedures there was not any certain moral problem. The patients' information was kept confidential.

Given the formula of sample size calculations in prevalence studies, $\left(\mathrm{N}=\mathrm{z}^{2} \times \mathrm{r} / \mathrm{d}^{2}, \mathrm{z}\right.$ and $\mathrm{r}$ were considered 1.96 and 0.03 , respectively (according to the references) and $a$ $=0.05$ and $0.01 \%$ difference in prevalence rate, the sample size of 134 people was gained for each group. Thus, the total sample size was estimated 268 people. Accordingly, a total of pregnant women were analyzed as follows: diabetes-free $(n=135)$, with gestational diabetes $(n=99)$ and with diabetes mellitus $(n=35)$.

The inclusion criteria of the study were: pregnant women at 24-34 weeks of gestation, singleton pregnancy, and necessary information in patients' medical case and accessibility to contact information of newborns by 6 weeks after birth.

The exclusion criteria of the study were multiple pregnancies, congenital anomalies, high-risk pregnancies (e.g. severe pre-eclampsia and eclampsia), placental complications, fetal growth restriction and polyhydramnios. In this study, women, in their 24-34 weeks of gestation, with PPROM were studied. Relying on the available information, the pregnant women of the study were divided into three groups, with diabetes mellitus (refer to definitions), gestational diabetes (refer to definitions) and diabetes-free; the diabetes-free group was matched with two other groups in terms of age of gestation and gravidity. Both maternal consequences including infection, chorioamnionitis, bleeding, need to episiotomy, vaginal birth or a surgical delivery by Caesarean section, manual placenta removal, bleeding after the childbirth (lochia), necessity of ICU admission, blood injection, stay period and fetal/ neonatal consequences including baby birth information, e.g., weight and age, Apgar score, infection, infant respiratory distress syndrome, need to revival, hypoglycemia, neonatal jaundice, need to NICU admission, stay period, need to insulin and death were analyzed and compared through all three groups.

It is necessary to remind that in all three groups, necessary information about baby's condition in terms of consequences/death were gained by 6 weeks after birth and they were considered as the neonatal consequences.

\section{Statistical Analysis}

The gained information was posited as standard deviation (SD), mean and frequency (percentage).

SPSS $^{\mathrm{TM}}$ version 16, was used in this study. Kolmogorov-Simonov test was used to analyze normal distribution of the quantitative data. One-way Analysis of variance (ANOVA) along with Tukey test were used to compare the quantitative variables. The qualitative data were compared between the two group using chi-square or Fisher exact test. $P<0.05$ was considered significant statistically.

\section{Results}

A total of 269 patients were studied through three groups: diabetes-free $(n=135)$, with gestational diabetes $(n=99)$ and with diabetes mellitus $(n=35)$. No significant statistical difference was found among the three groups in terms of the average interval between rupture of membrane and hospital admission $(P=0.35)$, average gestational age $(P=0.36)$.

The hospital stay period before childbirth was significantly different among three groups $(P=0.05)$ and the difference was significant between diabetes-free and gestational diabetes groups $(P=0.05)$; with the average hospital stay period was shorter in the diabetes-free group.

There was a significant statistical difference among the three groups in terms of the interval between admission and childbirth $(P=0.04)$ and this difference was only significant between diabetes-free group and gestational diabetes $(P=0.04)$, as the average interval between admission and childbirth was shorter in the diabetes-free group (Table 1).

As the results of this study indicate there were no sig-

Table 1. Interval Between Rupture of Membrane and Hospital Admission, Between Admission and Childbirth, Hospital Stay Period Before Childbirth, and the Average Gestational Age

\begin{tabular}{|c|c|c|c|c|c|}
\hline & $\begin{array}{l}\text { Case } \\
\text { numbers }\end{array}$ & $\begin{array}{l}\text { The average interval between } \\
\text { rupture of membrane and } \\
\text { hospital admission }\end{array}$ & $\begin{array}{l}\text { The hospital stay } \\
\text { period before } \\
\text { childbirth }\end{array}$ & $\begin{array}{l}\text { The average } \\
\text { gestational age(week) }\end{array}$ & $\begin{array}{l}\text { The interval } \\
\text { between admission } \\
\text { and childbirth(day) }\end{array}$ \\
\hline Diabetes-free & 135 & $0.44 \pm 0.10(0-8)$ & $4.16 \pm 0.47(1-33)$ & $32.49 \pm 2.01(25-34)$ & $2.06 \pm 0.36(1-24)$ \\
\hline Gestational diabetes & 99 & $0.57 \pm 0.22(0-14)$ & $6.13 \pm 0.73(1-43)$ & $32.09 \pm 2.51(25-34)$ & $3.75 \pm 0.61(1-29)$ \\
\hline Diabetes mellitus & 35 & $0.11 \pm 0.07(0-2)$ & $4.43 \pm 1.02(1-27)$ & $32.51 \pm 2.02(27-34)$ & $2.49 \pm 0.89(1-23)$ \\
\hline Meaningfulness & & 0.35 & 0.05 & 0.36 & 0.04 \\
\hline
\end{tabular}


nificant differences between the three groups in terms of number of chorioamnionitis cases $(P=0.20)$, more than $500 \mathrm{~mL}$ bleeding after the childbirth $(P=0.07)$, childbirth using Cesarean section $(P=0.07)$, vaginal delivery with induction $(P=0.07)$, the average gravidity (p:0.08), the average duration of the active phase of vaginal delivery $(P=0.12)$, the average child age $(P=0.58)$, the average apgar score of the first minute (p:0.16), the average Apgar score of the fifth minute (p:0.95) based on results of chisquare test and one-way ANOVA (Table 2).

The average duration of the latent phase of childbirth showed a significant difference between the three groups $(P=0.001)$. This difference was significant only between diabetes-free and gestational diabetes groups $(P=0.001)$, as the average duration of the latent phase of the vaginal childbirth was shorter in diabetes-free group.

A significant difference was found among the three groups in terms of the average interval between rupture of membrane and childbirth $(P=0.04)$. This difference was significant only between the diabetes-free group and gestational diabetes group $(P=0.04)$; as the average interval between membrane rupture and childbirth was shorter in the diabetes-free group.

Manual removal of placenta occurred in one case (1\%) of the gestational group. There was no need to ICU admission in diabetes-free group; while it was observed the gestational and mellitus groups two (2\%) and one (2.1\%) cases, respectively.

Chorioamnionitis for a patient and severe pre-eclampsia for two patients were reported as the reason for ICU admission. Need to injection of blood byproducts occurred only in one case (1\%) in the gestational diabetes group. Packed red blood cells were injected in this case.

Dystocia was reported only in a case (1\%) in the gestational diabetes group No newborn need insulin injection.

No significant difference in terms of consequences or death was observed within six weeks postnatal follow-up $(P=0.61)$. The most common reason for cesarean section

Table 2. Evaluation of Various Factors

\begin{tabular}{|c|c|c|c|c|}
\hline & Diabetes-Free & Gestational Diabetes & Diabetes Mellitus & Meaningfulness \\
\hline Chorioamnionitis & 3 case $(2.2 \%)$ & 4 case $(4 \%)$ & 3 case $(8.6 \%)$ & 0.20 \\
\hline More than $500 \mathrm{~mL}$ bleeding after the childbirth & 73 case $(54.1 \%)$ & 67 case $(67.5 \%)$ & 24 case $(68.6 \%)$ & 0.07 \\
\hline Childbirth using Cesarean section & 70 case $(51.9 \%)$ & 65 case $(65.7 \%)$ & 3 case $(2.2 \%)$ & 0.07 \\
\hline Vaginal delivery with induction & 14 case $(21.5 \%)$ & 21 case $(2.2 \%)$ & 1 case $(8.3 \%)$ & 0.07 \\
\hline The average gravidity & $2.04 \pm 0.9(1-6)$ & $1.86 \pm 0.67(1-6)$ & $2.20 \pm 1.02(1-6)$ & 0.08 \\
\hline $\begin{array}{l}\text { The average duration of the latent phase of vaginal } \\
\text { delivery (hour) }\end{array}$ & $3.79 \pm 1.71(1-6)$ & $3.69 \pm 1.55(1-6)$ & $2.75 \pm 0.75(1-6)$ & 0.12 \\
\hline The average child age (week) & $32.78 \pm 1.81$ & $32.54 \pm 2.02(26-30)$ & $32.80 \pm 1.75(28-34)$ & 0.58 \\
\hline $\begin{array}{l}\text { The average interval between rupture of membrane } \\
\text { and childbirth (hour) }\end{array}$ & $60.17 \pm 11.08(1-673)$ & $106.40 \pm 16.82(2-672)$ & $64.50 \pm 21.90(1-504)$ & 0.04 \\
\hline The average Apgar score of the first minute & $8.27 \pm 1.11(4-9)$ & $7.97 \pm 1.28(3-9)$ & $8.21 \pm 1.07(5-9)$ & 0.16 \\
\hline The average Apgar score of the fifth minute & $9.39 \pm 0.95(5-10)$ & $9.41 \pm 0.81(7-10)$ & $9.44 \pm 0.70(8-10)$ & 0.95 \\
\hline Infection & 1 case $(0.7 \%)$ & 2 case $(2 \%)$ & - & - \\
\hline Respiratory distress & 83 case $(61.5 \%)$ & 66 case $(66.7 \%)$ & 26 case $(74.3 \%)$ & 0.34 \\
\hline Need to revive newborns & 27 case $(20.0 \%)$ & 30 case $(30.3 \%)$ & 8 case $(22.9 \%)$ & 0.19 \\
\hline Neonatal jaundice & 65 case $(41.1 \%)$ & 48 case $(48.5 \%)$ & 42 case $(42.9 \%)$ & 0.83 \\
\hline Need to NICU admission & 105 case $(77.8 \%)$ & 76 case $(76.8 \%)$ & 28 case $(80.0 \%)$ & 0.93 \\
\hline Duration of neonatal hospital stay(day) & $6.27 \pm 0.83(1-57)$ & $8.04 \pm 1.12(1-48)$ & $6.07 \pm 1.61(1-46)$ & \\
\hline Hypoglycemia & 3 case & 5 case & 5 case & 0.01 \\
\hline
\end{tabular}

Table 3. Summary of the Reasons for Performing Cesarean Section Surgery in 3 Group

\begin{tabular}{|c|c|c|c|}
\hline \multirow[b]{2}{*}{ Cause } & \multicolumn{3}{|c|}{ Group } \\
\hline & $\begin{array}{c}\text { Diabetes-Free } \\
\text { No. (\%) }\end{array}$ & $\begin{array}{c}\text { Gestational Diabetes } \\
\text { No. (\%) }\end{array}$ & $\begin{array}{c}\text { Diabetes Mellitus } \\
\text { No. (\%) }\end{array}$ \\
\hline The history of cesarean section & $31(43.7)$ & $22(34.3)$ & $12(52.2)$ \\
\hline Placenta previa & $1(1.4)$ & $2(3.1)$ & $0(0)$ \\
\hline Active-phase arrest & $3(4.2)$ & $9(14.1)$ & $1(4.3)$ \\
\hline Narrow pelvic bone & $0(0)$ & $5(7.8)$ & $0(0)$ \\
\hline Fetal distress & $4(5.6)$ & $4(6.3)$ & $1(4.3)$ \\
\hline Pre-eclampsia & $4(5.6)$ & $1(1.6)$ & $3(13)$ \\
\hline Fetal presentation & $26(36.6)$ & $17(26.6)$ & $4(17.4)$ \\
\hline Meconium & $0(0)$ & $4(6.3)$ & $2(8.7)$ \\
\hline Colporrhaphy & $2(2.8)$ & $0(0)$ & $0(0)$ \\
\hline
\end{tabular}


Table 4. The Results of 6 Weeks Postnatal Follow-up for 3 Groups

\begin{tabular}{lccc}
\hline & & Group & \\
\cline { 2 - 4 } & Diabetes-Free (135 Cases) & Gestational Diabetes (99 Cases) & Diabetes Mellitus (35 Cases) \\
\hline Heart disease & $0(0 \%)$ & $1(20 \%)$ & $1(100 \%)$ \\
Seizure & $0(0 \%)$ & $1(20 \%)$ & $0(0 \%)$ \\
Death & $3(100 \%)$ & $3(60 \%)$ & $0(0 \%)$ \\
\hline
\end{tabular}

in three groups was previous cesarean section (Table 3 ).

Table 4 summarizes the results of 6 weeks postnatal follow-up for three groups. It is necessary to note that besides items included in this table, the following items have been recorded in a number of follow-ups whose cases belonged to past years:

Among children of 99 mothers with the gestational diabetes, a dysarthria (after 48 months) and a pulmonary hypertension (after 12 months) were reported. For children of 135 non-diabetic mothers difficulty in walking and growth retardation (after 12 months) were observed.

\section{Discussion and Conclusion}

As a clinical complication, gestational diabetes has been considered within past 50 years (6). The disease is associated with a high risk of mellitus diabetes type 2 during their lifetime $(6,9)$. The initial studies have shown that any failure to treat impaired carbohydrates tolerance during pregnancy will be followed by a high rate of mother's morbidity and her child's morbidity and mortality (7-9). This study aims at determining and analyzing gestational (maternal and neonatal) consequences in pregnant women with the diabetes mellitus and gestational diabetes and the control group.

A few studies have so far dealt with the relationship between mellitus/gestational diabetes and PPROM occurrence. Köck et al (10) studied about pregnancy retrospectively through two groups including pregnant women with former gestational diabetes and/or diabetes mellitus and normal pregnant women; their results showed that the potential of development of PPROM in first group was significantly more than that in the second group.

In India, Bhat et al (11) studied 286 pregnant women with gestational diabetes and 292 pregnant women free from (mellitus or gestational) diabetes in terms of PPROM occurrence. Their results indicated that the risk of PPROM in diabetes group was considerably higher than that in the normal group (more risk by 1.7 times).

In Oman, Al Riyami et al (12) studied 44 pregnant women with PPROM in terms of risk factors that may bring about this disorder. A significant relationship was reported between PPROM occurrence and gestational diabetes.

As the results of our study suggest, the only significant difference between the groups in question was shorter stay period of mothers and faster procedure of vaginal childbirth in the control group in comparison to the gestational diabetes group and also more cases with neonatal hyperglycemia in the group of diabetes mellitus in comparison to the control group; whereas no difference was found between the three groups in terms of other variables such as chorioamnionitis, bleeding after childbirth, Cesarean, need to induction, need to ICU/NICU admission, manual removal of placenta, Apgar, neonatal infection, respiratory distress, need to revive newborns, neonatal jaundice, shoulder dystocia, newborn's stay period, maternal/neonatal death.

Various studies about the relationship between simultaneous occurrence of diabetes and PPROM and maternal/ neonatal consequences were rare and incompatible:

Hollingsworth et al (13) indicated that the gestational diabetes has a significant relationship with the PPROM occurrence and also neonatal sepsis; whereas, our results, unlike Hollingworth et al (13), showed that there is no significant relationship between neonatal sepsis and infection and PPROM occurrence in the diabetic women rather the women in the control group. Magee et al (14) implied that hypoglycemia is the most prevalent complication of the gestational diabetes in women.

Boriboonhirunsarn et al (15) also reported neonatal hypoglycemia as the most common neonatal complication related to the maternal diabetes. Likewise, Bhat et al (11) reported that the neonatal hypoglycemia in patients with the gestational diabetes was significantly higher than that in patients in the control group.

As it is seen, findings of the three mentioned studies were compatible with ours in terms of finding a relationship between PPROM and maternal diabetes and neonatal hypoglycemia. However, it is necessary to say that this relationship in our study was only found in the diabetes mellitus rather the gestational diabetes. Although, it is not possible to point to a certain reason for this, the more chronic nature of diabetes mellitus in contrast to the gestational diabetes can be effective in this regard.

In their study, Ramírez (16) suggested that the gestational diabetes increases both the infection risk and the PPROM risk considerably (16). Sheiner et al (17) also demonstrated that there is a direct and significant relationship between asymptomatic bacteriuria during pregnancy and diabetes mellitus and PPROM; whereas no difference with the control group was seen in other cases (other unpleasant gestational consequences). In another study, Kessous et al (18) showed that there is a relationship between bacteriuria with streptococcus group B during pregnancy and chorioamnionitis. At the same time, it was shown that this type of infection is higher significantly in patients with the diabetes mellitus rather women without it. In other words, the diabetes mellitus in such patients has increased risk of chorioamnionitis in parallel with the increased risk of bacteriuria.

The results of the three mentioned studies were incompatible with ours in terms of the relationship between PPROM and diabetes and occurrence of the maternal in- 
fection. It may be due to some preventive measures taken in our medical centers, because various protocols have been used in different studies and as a result some variable consequences were reported.

Hamza et al (19) found a direct relationship between gestational diabetes and polyhydramnios in women with PPROM; with the gestational diabetes was associated with the risk of polyhydramnios leading to PPROM. In our study some significant statistical differences were found in the average hospital stay period before childbirth, the average interval between admission and childbirth, the average latent phase length of the vaginal childbirth and the average interval between rupture of membrane and childbirth in control group in contrast to women with gestational diabetes. They would be justified with the polyhydramnios associated with the diabetic mothers' pregnancy, which in turn increases the potential of PPROM; however, since our study is a retrospective one and there was no access to sonographies of pregnancy, it was not possible to examine the polyhydramnios in this study.

In Kuwait, Diejomaoh et al (20) studied 177 pregnant women with the diabetes mellitus. Eight percent of the patients had PPROM, who showed further pregnancy complications.

Tandu-Umba et al (21) studied 2086 pregnant women to analyze risk factors related to unpleasant (maternal-neonatal) pregnancy consequences. Both PPROM and diabetes mellitus were found major risk factors in this regard. Zhang et al (22) studied 604 women with gestational diabetes. They concluded that firstly, the potential of PPROM in this group of patients is increased and secondly, the risk of occurrence of unpleasant prognosis in this sort of pregnancies will be increased considerably.

It is necessary to remind that, as far as the available information resources show, our study is the first study on comparing the effect of simultaneous gestational/mellitus diabetes and PPROM on occurrence of maternal/neonatal consequences separately and in comparison with the control group. It seems that controlling glucose level plays a key role in PPROM development and pregnancy complications.

Boriboonhirunsarn et al (15) demonstrated that if diabetes is controlled properly, the potential of both maternal and neonatal consequences will be declined considerably.

$\mathrm{Yu}$ et al (23) the certain diet of diabetic patients decreased PPROM risk in pregnant women considerably. However, more controlled studies are needed to reach definite and final results on this area.

Finally, both diabetes mellitus and gestational diabetes are amongst the most common endocrine glands' anomalies which can affect negatively the childbirth during the gestation phase (20).

Marcello et al studied this effect for the first in 1882; they selected 22 pregnant women with diabetes. The study made it clear that only children of ten women were born alive. Researchers found the prevalence rates of abortion and preterm birth as $30 \%$ and $51 \%$ of the pregnant women with diabetes experienced intrauterine fetal death (24).
However, thanks to eye-catching advances in maternal and fetal care, currently the results of pregnancy are in diabetic and normal women. Here the most significant concerns are fetal overgrowth, traumas during childbirth, fetal macrosomia and other neonatal and maternal complications, especially eclampsia. Likewise, the clear increased prevalence of diabetes type 2 is expected in women with gestational diabetes and their children; as it is estimated that half of these women will suffer from diabetes within 20 coming years. Also, a strong relation was found between the gestational diabetes and their children's obesity (25).

Our results confirm this considerable difference in the results of taking care of diabetic pregnant mothers in the modern time rather the initial studies made already in this area.

On the other hand, Landon et al (26) indicated that although treating the gestational diabetes, in which glucose tolerance test is disturbed but fasting blood sugar test is normal, decreases the chance of pre-eclampsia, but in return it had no effect on other neonatal consequences including hyperbilirubinemia and intrauterine fetal death.

Therefore, in future studies, long-term follow-ups of mothers and newborns can be considered and the effect of treatment considering various conditions of patients can be considered.

Since this study in fact was conducted in order to find an answer for this question: Is it possible to use an identical protocol for women with gestational or mellitus diabetes and normal women in the case of PPROM until 34 weeks of the gestation or complications of pregnancy in this certain group will restrain this waiting period? Thus in our study, since the maternal and neonatal complications were not exacerbated, it is possible to continue pregnancy by 34 weeks of pregnancy through conducting PPROM protocol without any further risk and the same pregnancy termination indications can be used in people with PPROM and without diabetes.

\section{Ethical Issues}

The study was confirmed by the Ethics Committee of Tabriz Medical Sciences University.

\section{Conflict of Interests}

None.

\section{Financial Support}

This study was supported by researchers.

\section{References}

1. Martin RJ, Fanaroff AA, Walsh MC. Fanaroff and Martin's neonatal-perinatal medicine: diseases of the fetus and infant. Elsevier Health Sciences; 2014.

2. Polin RA, Spitzer AR. Fetal and neonatal secrets. Elsevier Health Sciences; 2013.

3. Jain V, Fisk NM. The twin-twin transfusion syndrome. Clin Obstet Gynecol 2004;47:181-202.

4. van Teeffelen AS, van der Ham DP, Willekes C, et al. Midtrimester preterm prelabour rupture of membranes 
(PPROM): expectant management or amnioinfusion for improving perinatal outcomes (PPROMEXILIII trial). BMC Pregnancy Childbirth 2014;14:128. doi:10.1186/1471-2393-14-128.

5. Mozurkewich E. Management of premature rupture of membranes at term: an evidence-based approach. Clin Obstet Gynecol. 1999;42:749.

6. O'Sullivan JB. Diabetes mellitus after GDM. Diabetes.1991;40 Suppl 2:131-135.

7. Kim C, Newton KM, Knopp RH. Gestational diabetes and the incidence of type 2 diabetes: a systematic review. Diabetes Care. 2002;25:1862-1868.

8. Kjos SL, Peters RK, Xiang A, Henry OA, Montoro M, Buchanan TA. Predicting future diabetes in Latino women with gestational diabetes. Utility of early postpartum glucose tolerance testing. Diabetes. 1995;44:586-591.

9. Damm P, Kuhl C, Bertelsen A, Molsted-Pedersen L. Predictive factors for the development of diabetes in women with previous gestational diabetes mellitus. Am J Obstet Gynecol. 1992;167:607-616.

10. Köck K, Köck F, Klein K, Bancher-Todesca D, Helmer H. Diabetes mellitus and the risk of preterm birth with regard to the risk of spontaneous preterm birth. J Matern Fetal Neonatal Med. 2010;23(9):1004-1008. doi:10.3109/14767050903551392.

11. Bhat M, Ramesha KN, Sarma SP, Menon S, Ganesh Kumar S. Outcome of gestational diabetes mellitus from a tertiary referral center in South India: a case-control study. J Obstet Gynaecol India. 2012;62:644-649. doi:10.1007/ s13224-012-0226-9.

12. Al Riyami N, Al-Ruheili I, Al-Shezaw F, Al-Khabori M. Extreme preterm premature rupture of membranes: risk factors and feto maternal outcomes. Oman Med J. 2013;28: 108-11. doi: 10.5001/omj.2013.28.

13. Hollingsworth DR, Vaucher Y, Yamamoto TR. Diabetes in pregnancy in Mexican Americans. Diabetes Care. 1991;14:695-705.

14. Magee MS, Walden CE, Benedetti TJ, Knopp RH. Influence of diagnostic criteria on the incidence of gestational diabetes and perinatal morbidity. JAMA. 1993;269:609615.

15. Boriboonhirunsarn D, Talungjit P, Sunsaneevithayakul P. Adverse pregnancy outcomes in gestational diabetes mellitus. J Med Assoc Thai. 2006;89:S23-S28.
16. Ramírez TM. [Gestational diabetes mellitus. Experience at a third level hospital]. Ginecol Obstet Mex. 2005;73(9):48491. 2005;73:484-491.

17. Sheiner E, Mazor-Drey E, Levy A. Asymptomatic bacteriuria during pregnancy. J Matern Fetal Neonatal Med. 2009;22:423-7. doi:10.1080/14767050802360783.

18. Kessous R, Weintraub AY, Sergienko R, et al. Bacteruria with group-B streptococcus: is it a risk factor for adverse pregnancy outcomes? J Matern Fetal Neonatal Med. 2012;25(10):1983-6. doi:10.3109/14767058.2012.671872.

19. Hamza A, Herr D, Solomayer EF, Meyberg-Solomayer G. Polyhydramnios: causes, diagnosis and therapy. Geburtshilfe Frauenheilkd. 2013;73:1241-1246. doi:10.1055/s-0033-136016320.

20. Diejomaoh MF, Gupta M, Farhat R, Jirous J, Al-Jaber $\mathrm{M}$, Mohd AT. Intrapartum performance of patients presenting with diabetes mellitus in pregnancy. Med Princ Pract. 2009;18:233-238. doi:10.1159/000204356.

21. Tandu-Umba B, Mbangama MA, Kamongola KM, et al. Pre-pregnancy high-risk factors at first antenatal visit: how predictive are these of pregnancy outcomes? Int J Womens Health. 2014;6:1011.

22. Zhang Y, Wang ZL, Liu B, Cai J. Pregnancy outcome of overweight and obese Chinese women with gestational diabetes. J Obstet Gynaecol. 2014;34:662-665. doi:10.310 9/01443615.2014.920787.

23. Yu XY, Zhang H. [Effects of a nutritional liquid supplement designed for diabetes mellitus on postprandial glucose and pregnancy outcomes in patients with gestational diabetes mellitus]. Zhonghua yi xue za zhi 2013;93:3450-3453.

24. LeRoith D, Taylor SI, Olefsky JM. Diabetes Mellitus: A fundamental and Clinical Text. Lippincott Williams \& Wilkins; 2004.

25. Rice MM, Landon MB; Eunice Kennedy Shriver National Institute of Child Health and Human Development Maternal-Fetal Medicine Units (MFMU) Network. What we have learned about treating mild gestational diabetes mellitus. Semin Perinatol. 2016;40(5):298-302. doi:10.1053/j.semperi.2016.03.006.

26. Landon MB, Spong CY, Thom E, et al. A multicenter, randomized trial of treatment for mild gestational diabetes. N Engl J Med. 2009;361:1339-1348. doi:10.1056/ NEJMoa0902430.

Copyright (C) 2017 The Author(s); This is an open-access article distributed under the terms of the Creative Commons Attribution License (http://creativecommons.org/licenses/by/4.0), which permits unrestricted use, distribution, and reproduction in any medium, provided the original work is properly cited. 